\title{
Screening for hub genes and signaling pathways of CD8+ T cells in systemic lupus erythematosus using bioinformatics
}

\author{
Yuefeng $\mathrm{Wu}^{1, *}$ \\ ${ }^{1}$ Zhejiang University-University of Edinburgh Institute, Zhejiang University, Haining, Zhejiang, CHINA
}

\begin{abstract}
Systemic lupus erythematosus (SLE) is a chronic autoimmune disease, which can damage multiple organs. The adaptive immune system, including CD8+ T cells, plays an essential role in this disease. However, the pathogenesis of SLE remains unclear, and there is a lack of effective diagnosis and treatment methods for SLE. In particular, there has been little research on SLE biomarkers, which have been widely studied and used in cancer diagnosis and treatment. In this study, bioinformatics tools were used to screen for hub genes and signaling pathways involving CD8+ T cells in patients with SLE. This is the first determination of metabolic abnormalities in SLE CD8+ T cells using bioinformatics pathway enrichment analysis. The PPI network and MCC algorithm identified SKP2 as a potential biomarker for SLE.
\end{abstract}

\section{Introduction}

Systemic lupus erythematosus (SLE) is a chronic autoimmune disease characterized by tissue inflammation and multiple organ damage. The mechanism of SLE is complicated and remains to be elucidated. Recent research has reported connections between SLE and genetics, environmental factors, and hormones ${ }^{[1]}$.

The adaptive immune system, especially $\mathrm{T}$ cells, plays an essential role in this process. Both deficiencies in and alterations of T-cell signals influence their proliferation, cytokine production, and even their functions ${ }^{[3]}$. Because epigenetic modifications can impact $\mathrm{T}$ cells in patients with SLE, DNA sequences from their $\mathrm{T}$ cells have been studied. An ex vivo study showed that IFN-regulated gene promoters were hypomethylated, which may result in the SLE phenotype $^{[2]}$.

Apart from generalized lymphocytopenia, specific $\mathrm{T}$ cell populations have been another focus; $\mathrm{CD} 8+\mathrm{T}$ cells could predict prognosis in $\mathrm{SLE}^{[5]}$. Expansion of CD8+ T cells is associated with poor prognoses because these cells can damage tissue.

Early diagnosis and precise classification of SLE will aid clinical treatment and patient recovery. Currently, the diagnosis of SLE is based on chest pains, arthralgias, and headaches ${ }^{[6]}$, and to our knowledge, there is no diagnostic method based on gene expression detection. However, with the development of sequencing methods and bioinformatics techniques, biomarkers have been widely used and studied with high specificity and sensitivity for other diseases.

Herein, we used GEO data to compare CD8+ T cells from patients with SLE and healthy controls. The data was preprocessed and normalized, and differentially expressed genes (DEGs) were identified using the $\mathrm{R}$ software. Next, we performed GO and KEGG enrichment for the DEGs. Additionally, the online tools STRING and Cytoscape were used to identify hub genes which could be used as biomarkers. The signal pathways were helpful for explaining the presence of abnormal CD8+ T cells in patients with SLE. This analysis offers insight into the mechanism of SLE and provides a potential biomarker for its diagnosis.

\section{Material \& Methods}

\subsection{GEO data query and normalization}

The GEO data were downloaded from the GEO database (http://www.ncbi.nih.gov/geo) using the following selection criteria ${ }^{[8]}$ : (1) patient diagnosed with SLE; (2) must have CD8+ T-cell data; and (3) must have CD8+ T-cell data from healthy control subjects. The GSE55447 dataset was selected ${ }^{[7]}$. Forty-two SLE samples and 10 samples from healthy controls were collected.

The downloaded MINiML files were normalized by $\log 2$ transformation using the preprocessCore package in $\mathrm{R}^{[10]}$. The probe IDs were translated into gene symbols. Probes representing more than one gene were excluded. The removeBatchEffect function in the Limma package was used to remove the batch effect ${ }^{[9]}$.

\subsection{Differential expression genes analysis}

The differential expression of genes was analyzed using the Limma package (version 3.40.2) of the R software. The adjusted $\mathrm{P}$ value was calculated to correct for falsepositive results in the GEO datasets. The thresholds for differentially expressed genes were defined as "Adjusted

\footnotetext{
Corresponding author: flaviuswu@zju.edu.cn
} 
$\mathrm{P}<0.05$ and Log (Fold-Change) $>1$ or Log (FoldChange) $<-1$." The DEGs were visualized with the help of a volcano plot and a heatmap. The ClusterProfiler in $\mathrm{R}$ was used to analyze potential signaling pathways. Two methods were used: gene ontology (GO) functions and Kyoto Encyclopedia of Genes and Genomes (KEGG) ${ }^{[11]}$.

\subsection{PPI network construction and hub gene identification}

The DEGs were uploaded to the online tool STRING (http://string-db.org/) for protein-protein interaction (PPI) network construction. Next, the TSV file containing the PPI information was downloaded and opened in Cytoscape. CytoHubba was used to identify hub genes in the network. The Maximal Clique Centrality (MCC) algorithm was used for calculating.

\section{Results}

\subsection{Differential expression genes result}

From the GSE55447 dataset, we collected CD8+ T cells from 42 patients with SLE and 10 healthy controls. We identified 428 DEGs in total; 134 upregulated genes and 294 downregulated genes. A heatmap and volcano plot show the DEGs (Fig. 1a,b).

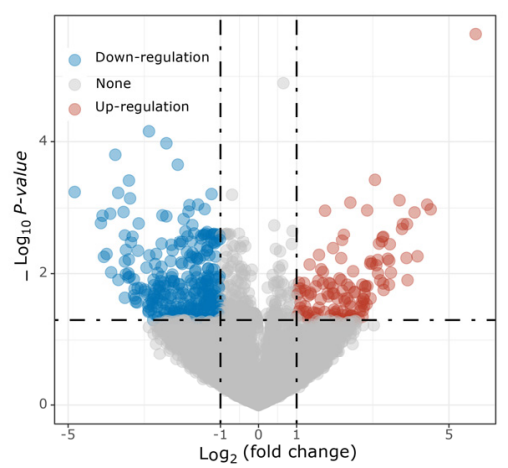

Fig. 1a Volcano plot of DEGs between CD8+ T cells from patients with SLE and healthy controls. The blue dots represent downregulated genes and the red dots represent upregulated genes.

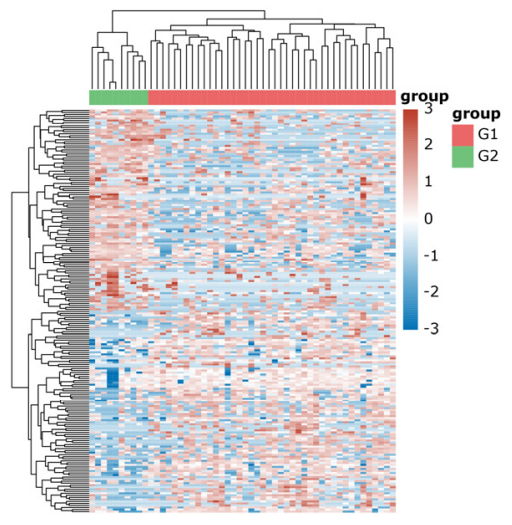

Fig. 1b Heatmap of DEGs between CD8 + T cells from patients with SLE and healthy controls. G1 is the SLE group and G2 is the control group. Colors represent how gene expression changed. The most significant 50 upregulated genes and 50 downregulated genes are shown here.

\subsection{DEGs function enrichment analysis}

The GO database is a widely used tool for systematically describing gene functions; it classifies genes into three groups: molecular function (MF), biological pathways (BP), and cellular components (CC ${ }^{[12]}$. In our analysis, many genes related to the processes of metabolism and biosynthesis were upregulated. Genes related to immunological processes were downregulated. The detailed information is listed in Fig. 2.

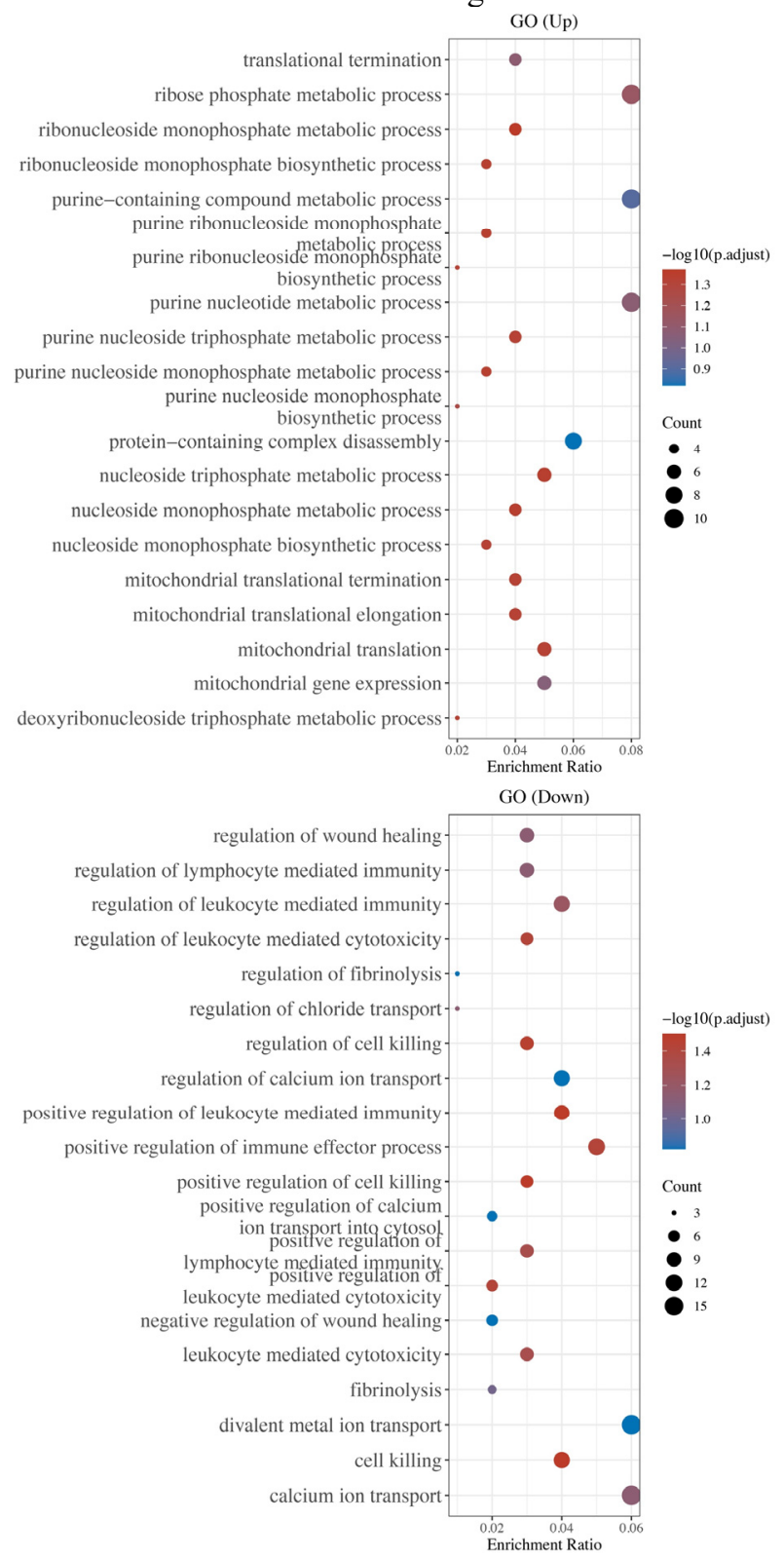

Fig. 2 The results of the GO term enrichment analysis, where different colors represent the significance of the enrichment results. The larger the number is, the lower the FDR will be. The size of the circle is representative of the number of genes. $\mathrm{P}<0.05$ or FDR $<0.05$ indicates a statistically significant pathway.

KEGG analysis can be used to present a more advanced view of the functional behavior of the cell[ ${ }^{[13]}$. KEGG enrichment results indicated that pathways related to biosynthesis, metabolism, hormones, and axons were upregulated. The JAK-STAT, cAMP, RIG-I-like receptor, GnRH, ErbB, and p53 signaling 
pathways were found to be downregulated, although their downregulation was not statistically significant. The details are listed in Fig. 3.

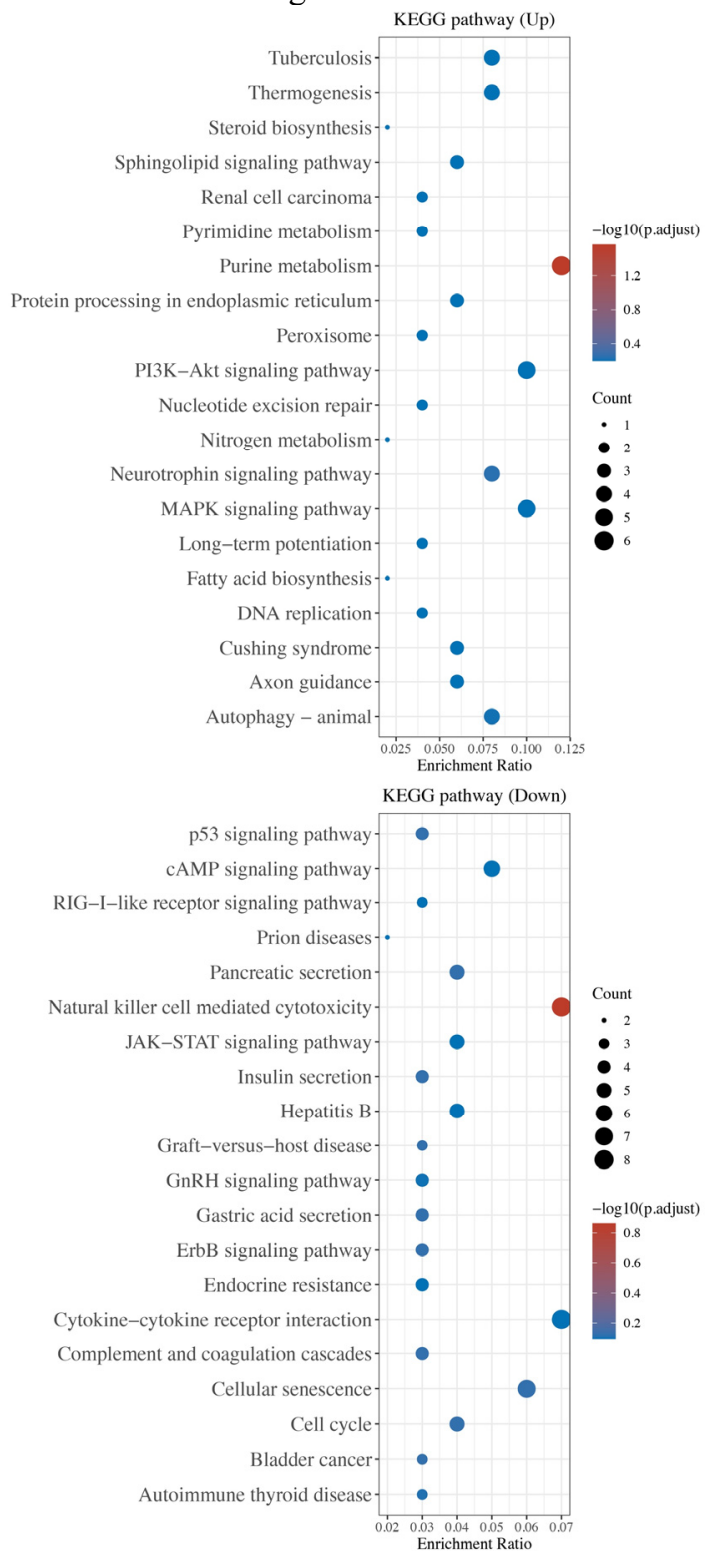

Fig. 3 KEGG enrichment results, in which different colors represent the level of significance of the enrichment results. The larger the number is, the lower the FDR will be. The size of the circle is representative of the number of genes. $\mathrm{P}<0.05$ or FDR $<0.05$ indicates a statistically significant pathway.

\subsection{PPI network construction and hub gene identification}

The interaction network generated by using STRING and visualized using Cytoscape contained 314 nodes and 617 edges (Fig. 5). Ten hub genes and their interactions were identified using MCC in cytoHubba (Fig. 4). The hub genes included $A S B 7, W S B 2, G R W D 1, C O P S 4$, FBXL13, KBTBD8, DCUN1D3, KLHL41, SKP2, and TULP4. SKP2 was the highest ranked hub gene. These genes were subjected to GO and KEGG analysis; the analysis indicated enrichment in protein metabolism modification.

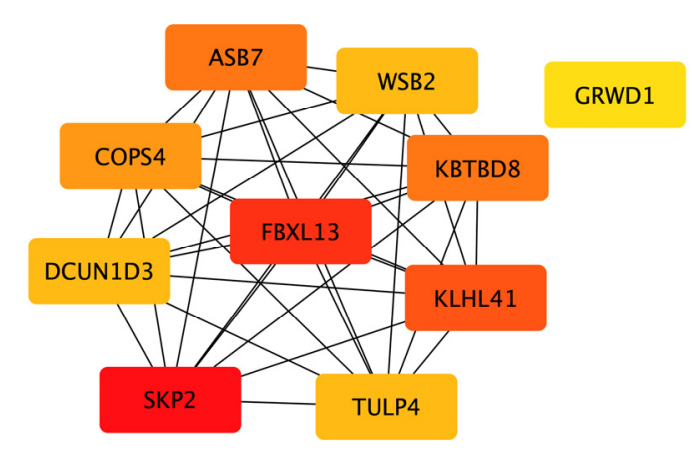

Fig. 4 Hub genes and their PPI network

\section{Discussion}

Early diagnosis and treatment of patients with SLE could improve their prognoses and remission rates. However, commonly used antibody testing cannot accurately predict these outcomes. Therefore, finding new biomarkers is increasingly important.

$\mathrm{T}$ cells play a critical role in SLE, and T-cell abnormalities have an essential impact on SLE pathogenesis. In our analysis, the metabolism of CD8+ T cells from patients with SLE differed from the CD8+ T cells of control subjects, which is consistent with previous reports ${ }^{[14]}$. However, it is the first use of bioinformatic analysis to reveal metabolic abnormalities in CD8 + T cells from patients with SLE and identify hub genes. The hyperexcitation of $\mathrm{T}$ cells may explain the symptoms of autoimmune disorders.

In line with our anticipation, the hub genes were enriched in pathways related to protein metabolism because T-cell hyperexcitation requires the adjustment of enzyme activities and more nutrients. Accumulating evidence has shown that $\mathrm{T}$-cell hyperexcitation is characterized by processes such as abnormal glycolysis, oxidative stress, and mitochondrial hyperpolarization ${ }^{[17]}$.

S-phase Kinase-associated Protein 2 (SKP2) controls the ubiquitination and subsequent proteasomal degradation of targeted cell cycle and signal transduction proteins ${ }^{[15]}$. It is also involved in the $\mathrm{G} 1 / \mathrm{S}$ transition, which makes it a critical target for cancer therapy ${ }^{[16]}$.

Moreover, the vitamin D receptor can suppress SLE by downregulating $S K P 2$, which provides support for recognition of $S K P 2$ as a biomarker in patients with SLE $^{[18]}$.

\section{Conclusion}

Our work identified the hub gene SKP2 in CD8+ T cells from patients with SLE as a potential biomarker for diagnosis or treatment. Moreover, bioinformatics analysis indicated that the metabolism of CD8 $+\mathrm{T}$ cells from patients with SLE differs from that in the CD8+ T cells of healthy controls, which provides insight for SLE treatment and may help explain the underlying mechanism of SLE. Moreover, this research also provided an insight into the way of enhance the $\mathrm{T}$ cell 
ability, which might be applied in the chimeric antigen receptor T-cell immunotherapy (CAR-T).

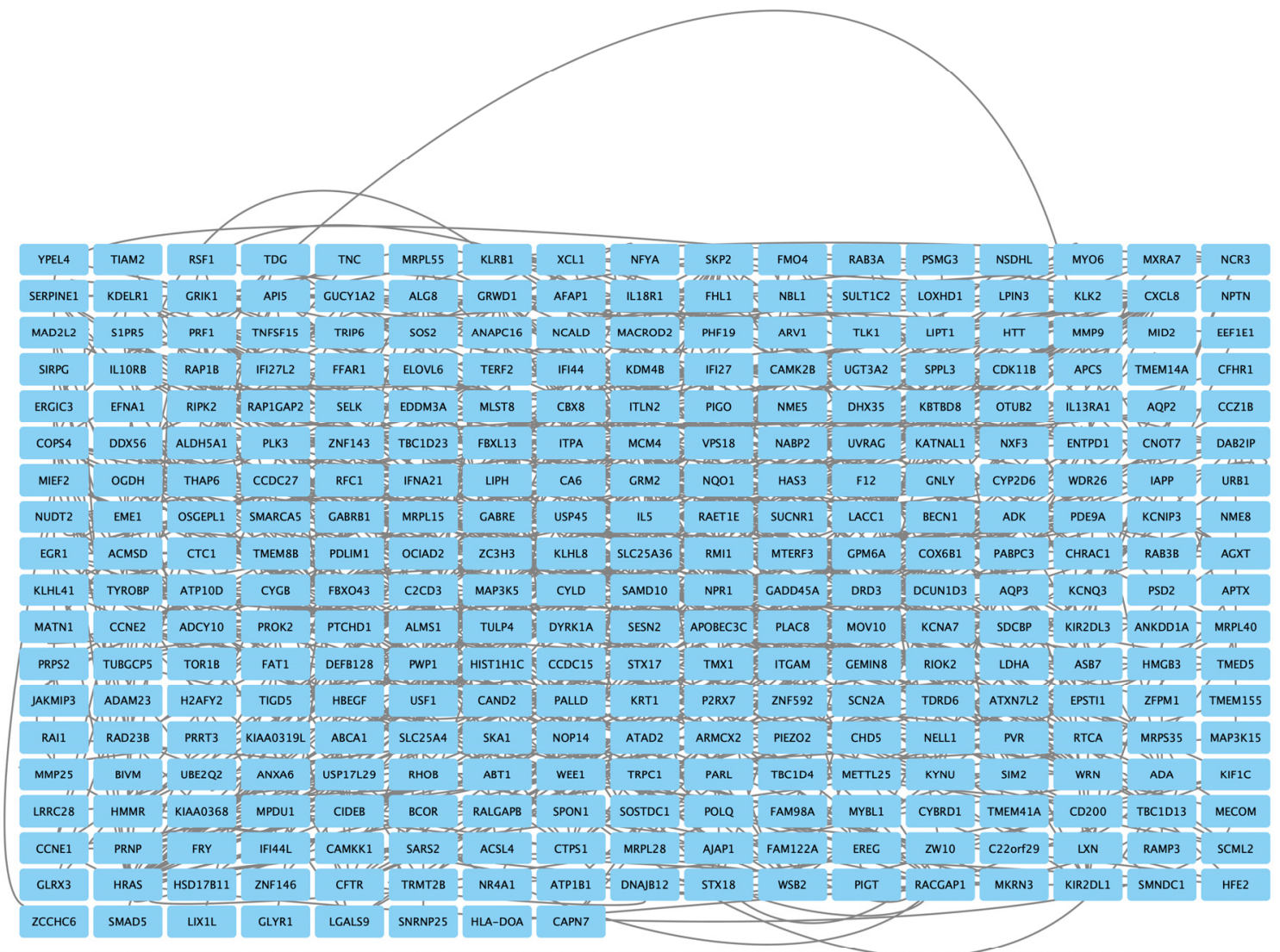

Fig. 5 PPI network for the predicted proteins of the identified differentially expressed genes (DEGs)

\section{References}

1. Katsuyama, Takayuki et al. "Aberrant $\mathrm{T}$ Cell Signaling and Subsets in Systemic Lupus Erythematosus." Frontiers in immunology vol. 9 1088. 17 May. 2018

2. Kaul A, Gordon C, Crow MK, Touma Z, Urowitz MB, van Vollenhoven R, Ruiz-Irastorza G, Hughes G. Systemic lupus erythematosus. Nat Rev Dis Primers. 2016 Jun 16;2:16039.

3. Crispín JC, Kyttaris VC, Terhorst C, Tsokos GC. T cells as therapeutic targets in SLE. Nat Rev Rheumatol. 2010 Jun;6(6):317-25. doi: 10.1038/nrrheum.2010.60. Epub 2010 May 11.

4. Coit P, Jeffries M, Altorok N, Dozmorov MG, Koelsch KA, Wren JD, Merrill JT, McCune WJ, Sawalha AH. Genome-wide DNA methylation study suggests epigenetic accessibility and transcriptional poising of interferon-regulated genes in naïve CD4+ $\mathrm{T}$ cells from lupus patients. J Autoimmun. 2013 Jun;43:78-84.

5. McKinney EF, Lyons PA, Carr EJ, Hollis JL, Jayne DR, Willcocks LC, Koukoulaki M, Brazma A, Jovanovic V, Kemeny DM, Pollard AJ, Macary PA, Chaudhry AN, Smith KG. A CD8+ T cell transcription signature predicts prognosis in autoimmune disease. Nat Med. 2010 May;16(5):586-91, 1p following 591.

6. Arnold, W. J. (ed.) American Rheumatism Association Glossary Committee: Dictionary of the Rheumatic Diseases. Vol I: Signs and Symptoms (American College of Rheumatology, 1982).

7. Sharma S, Jin Z, Rosenzweig E, Rao S, Ko K, Niewold TB. Widely divergent transcriptional patterns between SLE patients of different ancestral backgrounds in sorted immune cell populations. J Autoimmun. 2015 Jun;60:51-58. doi: 10.1016/j.jaut.2015.04.002. Epub 2015 Apr 24.

8. Barrett T, Wilhite SE, Ledoux P, Evangelista C, Kim IF, Tomashevsky M, Marshall KA, Phillippy KH, Sherman PM, Holko M, Yefanov A, Lee H, Zhang N, Robertson CL, Serova N, Davis S, Soboleva A. NCBI GEO: archive for functional genomics data sets--update. Nucleic Acids Res. 2013 Jan;41(Database issue):D991-5. doi: 10.1093/nar/gks1193. Epub 2012 Nov 27.

9. Ritchie, M.E., Phipson, B., Wu, D., Hu, Y., Law, C.W., Shi, W., and Smyth, G.K. (2015). limma powers differential expression analyses for RNA- 
sequencing and microarray studies. Nucleic Acids Research 43(7), e47.

10. Ben Bolstad (2021). preprocessCore: A collection of pre-processing functions. R package version 1.52.1. https://github.com/bmbolstad/preprocessCore

11. Yu G, Wang LG, Han Y, He QY. clusterProfiler: an $\mathrm{R}$ package for comparing biological themes among gene clusters. OMICS. 2012 May;16(5):284-7. doi: 10.1089/omi.2011.0118. Epub 2012 Mar 28.

12. Zhao Y, Wang J, Chen J, Zhang X, Guo M, Yu G. A Literature Review of Gene Function Prediction by Modeling Gene Ontology. Front Genet. 2020 Apr 24;11:400.

13. Kanehisa M. The KEGG database. Novartis Found Symp. 2002;247:91-101; discussion 101-3, 119-28, 244-52.

14. Morel L. Immunometabolism in systemic lupus erythematosus. Nat Rev Rheumatol. 2017 May;13(5):280-290.

15. Méndez J, Zou-Yang XH, Kim SY, Hidaka M, Tansey WP, Stillman B. Human origin recognition complex large subunit is degraded by ubiquitinmediated proteolysis after initiation of DNA replication. Mol Cell. 2002 Mar;9(3):481-91.

16. Cai Z, Moten A, Peng D, Hsu CC, Pan BS, Manne R, Li HY, Lin HK. The Skp2 Pathway: A Critical Target for Cancer Therapy. Semin Cancer Biol. 2020 Dec;67(Pt 2):16-33. doi: 10.1016/j.semcancer.2020.01.013. Epub 2020 Feb 1.

17. Sharabi A, Tsokos GC. T cell metabolism: new insights in systemic lupus erythematosus pathogenesis and therapy. Nat Rev Rheumatol. 2020 Feb;16(2):100-112.

18. Liu D, Fang YX, Wu X, Tan W, Zhou W, Zhang Y, Liu YQ, Li GQ. 1,25-(OH)2D3/Vitamin D receptor alleviates systemic lupus erythematosus by downregulating Skp2 and upregulating p27. Cell Commun Signal. 2019 Dec 10;17(1):163. 